\section{Influence of Plant Taxa on Pollinator, Butterfly, and Beneficial Insect Visitation}

\author{
Bethany A. Harris ${ }^{1}$ and S. Kristine Braman \\ Department of Entomology, 1109 Experiment Street, University of Georgia \\ Griffin Campus, Griffin, GA 30223
}

Svoboda V. Pennisi

Department of Horticulture, 1109 Experiment Street, University of Georgia Griffin Campus, Griffin, GA 30223

Additional index words. pollinating arthropods, flowering ornamental plants, biodiversity conservation, ecosystem services

\begin{abstract}
Insects provide ecosystem services, such as pollination and biological control. Additionally, flowering ornamental plant species have the potential to support beneficial insect communities, such as pollinating bees, wasps, and predatory plant bugs. We conducted visual observations and sampled via sweep nets to assess the potential of flowering ornamentals to act as a conservation resource for pollinators. Hoverflies (family: Syrphidae), small bees [Lasioglossum (dialictus) imitatum Smith and Halictus ligatus Say], skippers (family: Hesperiidae), predatory plant bugs (family Miridae), and parasitic wasps were frequent visitors to the specially designed Butterfly and Conservation Gardens. Agastache (Pursh) Kuntze 'Black Adder' and Celosia spicata L. were the most frequently visited by pollinators among 74 plant taxa. The results obtained will be useful in formulating recommendations on planting the best species for the purpose of attracting pollinators as well as for conservation purposes.
\end{abstract}

As decomposers, predators, and pollinators, insects play a vital role in ecosystem health, e.g., decomposers help aerate the soil, turning more soil than earthworms, and increasing soil rainwater retention and tillage (Pimentel, 2002). Predatory insects help keep the natural system in balance and prevent explosive pest population growth from taking over essential natural resources. There are over 6000 insect species that have been considered biological control agents and are being used to fight insect and weed pests (Pimentel, 2002). Moreover, countless other insect species act as pest population regulators but often go unnoticed. Insects also are a crucial part of food security in the United States, with 87 of the leading 115 food crops depending on insect pollinators (U.S. Fish and Wildlife Service, 2016).

Plants use a variety of characteristics, such as color, shape, and odor, to attract insects ensuring that these flower visitors

Received for publication 28 Apr. 2016. Accepted for publication 17 June 2016 .

This paper is a portion of a thesis submitted by Bethany A. Harris, in partial fulfillment of a master of science degree.

We thank Ellen Bauske and Paul Thomas for review of the manuscript and constructive criticism and Jerry Davis for his help with the statistical analyses. We also thank Georgia Master Gardener volunteers for their help with data collection. Technical assistance provided by Jim Quick, Brett Byous, Mary Sikora, Emma Brodzik, Alexia Maxis, and McKenzie Dallas was greatly appreciated.

1Corresponding author. E-mail: bah5191@uga.edu. will not leave without being dusted with pollen. Certain flower types are adapted for attracting particular insect groups, such as bees, butterflies, wasps, moths, beetles, and flies (Lázaro and Totland, 2014). Many plants depend on their mutualistic relationships with pollinating arthropods for survival. In turn, pollinators rely on flowering plants to acquire food including pollen and nectar (Kearns and Inouye, 1997).

Research shows that pollinators contribute to $35 \%$ of the world's food production and 24 billion dollars in the U.S. economy (Astegiano et al., 2015). Likewise, native pollinators, such as bumble bees, sweat bees, and leafcutter bees, provide crop benefits that are valued at more than 9 billion dollars (Astegiano et al., 2015). Evidence suggests that pollinating insects of many important plants are declining worldwide (Astegiano et al., 2015; Fukase and Simons, 2016; NRC National Research Council, 2007). Land use, loss, and fragmentation of habitat, modern agricultural practices, and pesticide use pose great threats to insect pollinators and natural enemies (Nicholls and Altieri, 2013). Since 2006, it has been reported that western honeybee (Apis mellifera L.) colony loss rates have increased to an average of $30 \%$ each winter due to a variety of factors including loss of natural forage and inadequate diets, mite infestation and diseases, loss of genetic diversity, and exposure to certain pesticides (Astegiano et al., 2015). Other native pollinators, including bumble bees and monarch butterflies (Danaus plexippus L.), have experienced population declines as well (NRC
National Research Council, 2007). Systemic pesticides used in production of agricultural products, including ornamental plants, as one of the factors contributing to declining pollinator health (Astegiano et al., 2015).

Providing essential floral resources to natural enemies and pollinating insects can increase biological control and pollination (Fiedler and Landis, 2007; Woltz et al., 2012). Research suggests a diverse butterfly population can be attained through the addition of annual, perennial, and woody shrub ornamental species to a landscape (Poythress and Affolter, 2015; Wilde et al., 2015). Several studies have noted that gardens provide resources through flowers as well as nesting areas that contribute to the survival and reproduction of bees (Frankie et al., 2005; Matteson et al., 2008; McIntyre and Hostetler, 2001). Likewise by attracting pollinators and beneficial insects, ecosystem services can also be attained.

By monitoring pollinator and beneficial insect occurrence within habitat management sites through the use of sweep-net sampling and visual observations, ornamental plant species can be evaluated for their arthropod attractiveness and the provision of arthropodmediated ecosystem services including pollination and biological control in southeastern landscapes (Kremen et al., 2007). The goal of this study was to provide detailed information on the attractiveness of a wide selection of ornamental floral resources to pollinators, natural enemies, and phytophagous insects as detailed and systematic assessment of pollinators and biological predators on plant species have been limited.

\section{Materials and Methods}

Research plots layout, soil preparation, plant selection, and plant maintenance. The research was conducted at the University of Georgia Griffin Campus, Griffin, GA $\left(33^{\circ} 24^{\prime} 67^{\prime \prime} \mathrm{N}, 84^{\circ} 26^{\prime} 40^{\prime \prime} \mathrm{W}\right)$. The research plots (hereafter referred to as "Butterfly and Conservation Gardens") were established in Oct. 2012. Four plots (total $1705.2 \mathrm{~m}^{2}$, $426.3 \mathrm{~m}^{2}$ per plot) were established in native soil (sandy clay, pH: 5.1,organic matter 3.25\%, carbon $0.16 \%$, nitrogen $0.04 \%$ ) and amended with mulch (West Fraser Timber, Opelika, AL) incorporated at the rate of $2.36 \mathrm{~m}^{3}$ per $92.9 \mathrm{~m}^{2}$ at a depth of $15.24-20.32 \mathrm{~cm}$. Soil samples were collected before planting. No fertilizer was applied prior or during the study, however, no visual symptoms of nutrient-related disorders were observed and plant growth did not appear adversely affected. A total of 74 commercially available annual and perennial herbaceous and shrub ornamentals, including exotic and native plant species, were used. Plants were either grown in-house or purchased from local vendors and at planting were $15.2-\mathrm{cm}, 3.8-\mathrm{L}$, and $11.3-\mathrm{L}$ containers for annual, herbaceous perennials, and woody shrubs, respectively. Plants were selected based on their attractiveness to pollinating insects (commercial marketing information and various web sources) and horticultural attributes 
and superior adaptability to the southeastern growing environment. Effort was made to create an aesthetically pleasing and seasonally attractive landscape which necessitated the use of various foliage textures and contrasts, e.g., fine vs. coarse, as well as a variety of plant habits, e.g., groundcover vs. upright. To this end, nonnative exotic species prized primarily for their colorful and/or bold foliage such as Colocasia, Hibiscus acetosella, Stachys, and gingers were planted. In addition, Foeniculum, Petroselinum, Melissa, and Passiflora also were incorporated in the planting to serve as food source for the larval stages of various insect species. No pesticides were used for pest control throughout the study.

There were four replicate plots within the garden, three were contiguous and separated by buffer pathways, while the fourth was located in $\approx 500 \mathrm{~m}$ away. Within each of the four plots, each plant species/cultivar had three sub samples. Irrigation was delivered by overhead sprinklers (Hunter Industries Controller with MP Rotator heads on risers, San Marcos, CA) and provided $2.5 \mathrm{~cm}$ of water weekly.

Monitoring of insect activity. Pollinator and beneficial insect activity was monitored using two methods: visual observations and sweep-net sampling.

Visual observations. Each of the four garden replicates was monitored using visual observations at 10:30-11:30 AM and 2:30-3:30 PM twice weekly from 4 June to 5 Sept. 2013. Each plant was observed for $2.0-3.0 \mathrm{~s}$ and observations recorded included family and order of insects viewed and plant or location the insect was found.

Sweep-net sampling. In 2014, sweep-net sampling was performed in the four garden replicates in which plant taxa were sampled with an aerial net (diameter: $30.5 \mathrm{~cm}$, handle length: $45.7 \mathrm{~cm}, 7112 \mathrm{NA}$; Bioquip Products, Rancho Dominguez, CA). The net was passed over the outer perimeter of the plant canopy of each plant five times, where the highest density of flowering stems was located). Sweep-net sampling occurred once a week from 10 June to 22 July 2014. Sweep-net contents were placed into a plastic bag and stored in a freezer for preservation until sorting, mounting, and insect identification occurred.

Statistical analysis. Data were subjected to analysis of variance using the general linear model procedure. Data were transformed before analysis using the arcsin square root transformation for percentage data and square root transformation for count data. Backtransformed data are being reported. For visual observations and sweep-net sampling, plant type was analyzed as a main effect. The main effects of the variables were examined but interactions were not included in the models. Main treatment means for visual observations and sweep-net sampling were separated using Tukey's honestly significant difference test (SAS Institute, 2010).

\section{Results}

Visual observations: Pollinators. Potential pollinators observed during visual observations consisted of bumble bees (Bombus sp. Latreille), small bees [i.e., Lasioglossum (dialictus) imitatum Smith and H. ligatus], other bees (i.e., Xylocopa sp. Latreille and A. mellifera), long-legged flies (Dolichopodidae), hover flies (Syrphidae), house flies (Muscidae), tumbling flower beetles (Mordellidae), skipper butterflies (Hesperiidae), cabbage butterflies (Pieridae), and the common buckeye butterfly (Junonia coenia Hübner) (Table 1). These groups were combined into a single category "All Pollinators." Plant taxa were constant, but the number of pollinators visiting each varied $(\mathrm{F}=8.44, \mathrm{df}=73,4391$, $P \leq 0.0001$; Table 2).

Of the plant types in the study, the 15 most often visited by pollinators were Agastache (Pursh) Kuntze 'Black Adder', Gaura lindheimeri Engelm 'Passionate Blush', Lonicera sempervirens L. 'Major Wheeler', Agastache mexicana (Pursh) Kuntze Acapulco ${ }^{\mathrm{TM}}$, Nepeta $\times$ faassenii Bergmans 'Walker's Low', Foeniculum vulgare L., Coreopsis auriculata L. 'Redshift', Aster dumosus L. 'Wood's Pink', Rudbeckia triloba L., Belamcanda chinensis L., Salvia microphylla Kunth 'Hot Lips', Solenostemon scrutellariodes Mariposa ${ }^{\mathrm{TM}}$ L., Salvia M. Martens and Galeotti 'Mystic Spires', Echinacea purpurea (L.) Moench 'Magnus', and Rudbeckia hirta L. 'Indian Summer' among other plant types (Table 2).

Visual observations: Beneficial insects. Potential beneficial insect taxa observed included robber flies (Asilidae), soldier beetles (Cantharidae), fireflies (Lampyridae), predaceous plant bugs (Miridae, i.e., Lygus sp. Hahn), predaceous stink bugs (Pentatomidae, i.e., Euthyrhynchus sp. L.), predaceous tree crickets (Oecanthinae), big-eyed bugs (Geocoris punctipes Say) (Table 3). These groups were combined into a single category "All Beneficial Insects." Plant taxa were constant, but the number of beneficial insects visiting each varied $(\mathrm{F}=6.18, \mathrm{df}=73,4380, P \leq$ 0.0001; Table 1).

Of the plant types observed, the 15 most often visited by beneficial insects were $G$.

Table 1. Influence of plant taxa on pollinator and beneficial insect taxa occurrence observed in the University of Georgia-Griffin Butterfly and Conservation Gardens $(n=4)$ (Spalding Co., GA).

\begin{tabular}{lccr}
\hline Insect type & df & $P$ value & F value \\
\hline Bumble bee & 73,4391 & $<0.0001^{* * *}$ & 13.14 \\
Long-legged fly & 73,4391 & $<0.0001^{* * *}$ & 4.87 \\
Skipper butterfly & 73,4391 & $<0.0001^{* * *}$ & 2.63 \\
Tumbling flower beetle & 73,4391 & $<0.0001^{* * *}$ & 8.74 \\
House fly & 73,4391 & $<0.0001^{* * *}$ & 3.32 \\
Other bee & 73,4391 & $<0.0001^{* * *}$ & 3.73 \\
Cabbage butterfly & 73,4391 & $<0.0001^{* * *}$ & 11.13 \\
Small bee & 73,4391 & $<0.0001^{* * *}$ & 6.00 \\
Hover fly & 73,4391 & $<0.0001^{* * *}$ & 2.89 \\
Buckeye butterfly & 73,4391 & $<0.0001^{* * *}$ & 2.61 \\
All Pollinators combined & 73,4391 & $<0.0001^{* * *}$ & 8.44 \\
Robber fly & 73,4391 & $<0.0001^{* * *}$ & 2.05 \\
Soldier beetle & 73,4391 & $<0.0001^{* * *}$ & 3.92 \\
Firefly & 73,4390 & $<0.0001^{* * *}$ & 4.37 \\
Predaceous plant bug & 73,4391 & $<0.0001^{* * *}$ & 8.74 \\
Predaceous tree cricket & 73,4391 & $<0.0001^{* * *}$ & 3.97 \\
Predatory stink bug & 73,4390 & $<0.0001^{* * *}$ & 2.08 \\
Big-eyed bug & 73,4391 & $<0.0001^{* * *}$ & 2.78 \\
All Beneficial Insects combined & 73,4389 & $<0.0001^{* * *}$ & 6.18 \\
\hline
\end{tabular}

$*, * *, * * *$ Significant at $P<0.05,0.01$, or 0.001 , respectively.

lindheimeri 'Passionate Blush', Achillea filipendulina Lamarck 'Coronation Gold', Buddleia davidii Franch 'Sweet Profusion', $C$. auriculata 'Redshift', Curcuma petiolata Roxburgh 'Emperor', L. sempervirens 'Major Wheeler', $R$. triloba, R. hirta 'Indian Summer', Cassia $\times$ floribunda (Cav.) Irwin and Barneby, Achillea millefolium L. 'Sunny Seduction', C. auriculata. 'Snowberry', F. vulgare, C. spicata, Coreopsis L. 'Cosmic Eye', and Stachys byzantina Koch among other plant types (Table 2).

Sweep-net sampling: Pollinators. Potential pollinators collected during sweep-net sampling consisted of bumble bees (Bombus sp. Latreille), small bees [i.e., Lasioglossum (dialictus) imitatum Smith and H. ligatus], carpenter bees (Xylocopa sp. Latreille), western honeybees ( $A$. mellifera $)$, organ pipe mud dauber (Trypoxylon politum Drury), longlegged flies (Dolichopodidae), house flies (Muscidae), hover flies (Syrphidae), picturewinged flies (Uliididae), tumbling flower beetles (Mordellidae), sphinx moths (Hemaris sp. Dalman), ermine moths (Yponomeutidae), fiery skipper butterflies (Hylephila phyleus Drury), and common buckeye butterflies (J. coenia) (Table 4). These groups were combined into a single category 'All Pollinators'. Plant taxa were constant, but the number of pollinators visiting each varied $(\mathrm{F}=3.27, \mathrm{df}=38,877, P \leq 0.0001$; Table 3).

Of the plant types sampled in 2014 , the 15 plants most frequently visited by pollinators were C. spicata, Lysimachia ciliata L. 'Firecracker', Amsonia hubrichtii Woodson, Nepeta ×faassenii 'Walker's Low', $R$. hirta 'Indian Summer', Rudbeckia fulgida var. sullivantii 'Goldsturm', A. millefolium 'Sunny Seduction', R. triloba, A. filipendulina 'Coronation Gold', Abelia $\times$ grandiflora (André) Rehd. 'Raspberry Profusion', B. chinensis, E. purpurea 'Magnus', Astilbe chinensis (Knoll) Maximowicz 'Visions in Pink', C. petiolata 'Emperor', and Colocasia esculenta 'Black Stem'
HortScience Vol. 51(8) August 2016 
Table 2. Mean $( \pm \mathrm{SE})$ of pollinators and other beneficial insects directly observed on plant types in the University of Georgia-Griffin Butterfly and Conservation Gardens $(n=4)$ (Spalding Co., GA).

\begin{tabular}{|c|c|c|}
\hline Plant name & All pollinators & All beneficial insects \\
\hline$\overline{A b e l i a} \times$ grandiflora 'Raspberry Profusion' & $0.20 \pm 0.06$ fghi & $0.00 \pm 0.00 \mathrm{~d}$ \\
\hline Achillea filipendulina 'Coronation Gold' & $0.28 \pm 0.09$ defghi & $0.38 \pm 0.09 \mathrm{a}$ \\
\hline Achillea millefolium 'Sunny Seduction' & $0.22 \pm 0.06$ efghi & $0.18 \pm 0.05$ abcde \\
\hline Agastache 'Black Adder' & $1.13 \pm 0.14 \mathrm{a}$ & $0.05 \pm 0.03 \mathrm{de}$ \\
\hline Agastache mexicana Acapulco ${ }^{\mathrm{TM}}$ & $0.78 \pm 0.24 \mathrm{abcd}$ & $0.05 \pm 0.03 \mathrm{de}$ \\
\hline Allium senescens ssp. glaucum & $0.00 \pm 0.00 \mathrm{i}$ & $0.00 \pm 0.00 \mathrm{e}$ \\
\hline Amsonia hubrichtii & $0.03 \pm 0.02 \mathrm{hi}$ & $0.08 \pm 0.04 \mathrm{cde}$ \\
\hline Aster dumosus 'Wood's Pink' & $0.55 \pm 0.26$ bcdefgh & $0.07 \pm 0.03 \mathrm{de}$ \\
\hline Aster laevis 'Bluebird' & $0.02 \pm 0.02 \mathrm{hi}$ & $0.05 \pm 0.03 \mathrm{de}$ \\
\hline Aster novae-angliae 'Professor Kippenburg' & $0.02 \pm 0.02 \mathrm{hi}$ & $0.00 \pm 0.00 \mathrm{e}$ \\
\hline Aster tataricus 'Jindai' & $0.00 \pm 0.00 \mathrm{i}$ & $0.00 \pm 0.00 \mathrm{e}$ \\
\hline Astilbe chinensis 'Visions in Pink' & $0.05 \pm 0.03 \mathrm{hi}$ & $0.03 \pm 0.02 \mathrm{de}$ \\
\hline Belamcanda chinensis & $0.45 \pm 0.24$ cdefghi & $0.00 \pm 0.00 \mathrm{e}$ \\
\hline Breynia nivosa & $0.00 \pm 0.00 \mathrm{i}$ & $0.00 \pm 0.00 \mathrm{e}$ \\
\hline Buddleia davidii 'Sweet Profusion' & $0.10 \pm 0.05$ fghi & $0.33 \pm 0.11 \mathrm{ab}$ \\
\hline Caryopteris $\times$ clandonensis 'First Choice' & $0.00 \pm 0.00 \mathrm{i}$ & $0.00 \pm 0.00 \mathrm{e}$ \\
\hline Cassia $\times$ floribunda & $0.08 \pm 0.04$ fghi & $0.18 \pm 0.06$ abcde \\
\hline Celosia spicata & $0.33 \pm 0.11$ defghi & $0.13 \pm 0.06$ bcde \\
\hline Cephalanthus occidentalis & $0.00 \pm 0.00 \mathrm{i}$ & $0.03 \pm 0.02 \mathrm{de}$ \\
\hline Ceratostigma plumbaginoides & $0.00 \pm 0.00 \mathrm{i}$ & $0.00 \pm 0.00 \mathrm{e}$ \\
\hline Chrysanthemum 'Cambodian Queen' & $0.00 \pm 0.00 \mathrm{i}$ & $0.00 \pm 0.00 \mathrm{e}$ \\
\hline Clethra alnifolia 'Hummingbird' & $0.00 \pm 0.00 \mathrm{i}$ & $0.07 \pm 0.03 \mathrm{de}$ \\
\hline Colocasia esculenta 'Black Stem' & $0.07 \pm 0.03 \mathrm{ghi}$ & $0.05 \pm 0.03 \mathrm{de}$ \\
\hline Coreopsis 'Cosmic Eye' & $0.32 \pm 0.11$ defghi & $0.13 \pm 0.06$ bcde \\
\hline Coreopsis auriculata 'Snowberry' & $0.32 \pm 0.11$ defghi & $0.18 \pm 0.06$ abcde \\
\hline Coreopsis auriculata 'Redshift' & $0.59 \pm 0.14$ bcdefg & $0.29 \pm 0.06 \mathrm{abc}$ \\
\hline Curcuma petiolata 'Emperor' & $0.27 \pm 0.07$ defghi & $0.22 \pm 0.07 \mathrm{abcd}$ \\
\hline Dianthus gratianopolitanus 'Firewitch' & $0.00 \pm 0.00 \mathrm{i}$ & $0.02 \pm 0.02 \mathrm{de}$ \\
\hline Dianthus hybrida 'Coconut Surprise' & $0.00 \pm 0.00 \mathrm{i}$ & $0.00 \pm 0.00 \mathrm{e}$ \\
\hline Dicliptera suberecta & $0.00 \pm 0.00 \mathrm{i}$ & $0.00 \pm 0.00 \mathrm{e}$ \\
\hline Digitalis purpurea 'Excelsior Hybrid Mix' & $0.00 \pm 0.00 \mathrm{i}$ & $0.00 \pm 0.00 \mathrm{e}$ \\
\hline Digitalis purpurea f. albiflora 'Alba' & $0.00 \pm 0.00 \mathrm{i}$ & $0.08 \pm 0.05 \mathrm{cde}$ \\
\hline Echinacea purpurea 'Magnus' & $0.37 \pm 0.12$ cdefghi & $0.02 \pm 0.02 \mathrm{de}$ \\
\hline Eupatorium purpureum ssp. maculatum 'Gateway' & $0.22 \pm 0.05$ efghi & $0.12 \pm 0.04$ bcde \\
\hline Eupatorium rugosum 'Chocolate' & $0.02 \pm 0.02 \mathrm{hi}$ & $0.00 \pm 0.00 \mathrm{e}$ \\
\hline Foeniculum vulgare & $0.62 \pm 0.13$ abcdef & $0.13 \pm 0.04$ bcde \\
\hline Gaura lindheimeri 'Passionate Blush' & $1.07 \pm 0.33 \mathrm{ab}$ & $0.40 \pm 0.08 \mathrm{a}$ \\
\hline Hedychium coronarium & $0.05 \pm 0.03 \mathrm{hi}$ & $0.02 \pm 0.02 \mathrm{de}$ \\
\hline Heliopsis helianthoides var. scabra 'Summer Sun' & $0.18 \pm 0.06$ fghi & $0.08 \pm 0.04$ cde \\
\hline Hibiscus acetosella Mahagony Splendor & $0.02 \pm 0.02 \mathrm{hi}$ & $0.00 \pm 0.00 \mathrm{e}$ \\
\hline Itea virginica & $0.05 \pm 0.03 \mathrm{hi}$ & $0.00 \pm 0.00 \mathrm{e}$ \\
\hline Lantana camara 'Mozelle' & $0.10 \pm 0.04$ fghi & $0.03 \pm 0.02 \mathrm{de}$ \\
\hline Lantana camara 'Ms. Huff' & $0.17 \pm 0.06$ fghi & $0.07 \pm 0.03 \mathrm{de}$ \\
\hline Lantana camara 'Pink Caprice' & $0.02 \pm 0.02 \mathrm{hi}$ & $0.03 \pm 0.02 \mathrm{de}$ \\
\hline Lonicera sempervirens 'Major Wheeler' & $0.88 \pm 0.30 \mathrm{abc}$ & $0.22 \pm 0.09$ abcd \\
\hline Lysimachia ciliata 'Firecracker' & $0.03 \pm 0.02 \mathrm{hi}$ & $0.02 \pm 0.02 \mathrm{de}$ \\
\hline Melissa officinalis & $0.00 \pm 0.00 \mathrm{i}$ & $0.00 \pm 0.00 \mathrm{e}$ \\
\hline Monarda didyma 'Raspberry Wine' & $0.05 \pm 0.03 \mathrm{hi}$ & $0.03 \pm 0.02 \mathrm{de}$ \\
\hline Nepeta $\times$ faassenii 'Walker's Low' & $0.74 \pm 0.14$ abcde & $0.02 \pm 0.02 \mathrm{de}$ \\
\hline Nicotiana alata & $0.00 \pm 0.00 \mathrm{i}$ & $0.00 \pm 0.00 \mathrm{e}$ \\
\hline Passiflora caerulea & $0.00 \pm 0.00 \mathrm{i}$ & $0.00 \pm 0.00 \mathrm{e}$ \\
\hline Petroselinum crispum & $0.00 \pm 0.00 \mathrm{i}$ & $0.03 \pm 0.03 \mathrm{de}$ \\
\hline Petunia $\times$ hybrid 'Fuseables' & $0.02 \pm 0.02 \mathrm{hi}$ & $0.00 \pm 0.00 \mathrm{e}$ \\
\hline Phlox paniculata 'Robert Poore' & $0.02 \pm 0.02 \mathrm{hi}$ & $0.02 \pm 0.02 \mathrm{de}$ \\
\hline Rudbeckia fulgida var. sullivantii 'Goldsturm' & $0.10 \pm 0.05$ fghi & $0.10 \pm 0.06 \mathrm{cde}$ \\
\hline Rudbeckia hirta 'Indian Summer' & $0.35 \pm 0.07$ cdefghi & $0.19 \pm 0.07$ abcde \\
\hline Rudbeckia triloba & $0.53 \pm 0.12$ cdefghi & $0.20 \pm 0.09$ abcde \\
\hline Ruellia brittoniana 'Purple Showers' & $0.02 \pm 0.02 \mathrm{hi}$ & $0.02 \pm 0.02 \mathrm{de}$ \\
\hline Ruellia brittoniana 'White Katie' & $0.00 \pm 0.00 \mathrm{i}$ & $0.00 \pm 0.00 \mathrm{e}$ \\
\hline Salvia 'Mystic Spires' & $0.38 \pm 0.13$ cdefghi & $0.00 \pm 0.00 \mathrm{e}$ \\
\hline Salvia chiapensis & $0.00 \pm 0.00 \mathrm{i}$ & $0.00 \pm 0.00 \mathrm{e}$ \\
\hline Salvia elegans & $0.08 \pm 0.04$ fghi & $0.00 \pm 0.00 \mathrm{e}$ \\
\hline Salvia leucantha 'Santa Barbara' & $0.00 \pm 0.00 \mathrm{i}$ & $0.00 \pm 0.00 \mathrm{e}$ \\
\hline Salvia microphylla 'Hot Lips' & $0.41 \pm 0.12$ cdefghi & $0.07 \pm 0.03 \mathrm{de}$ \\
\hline Setaria palmifolia 'Rubra Variegata' & $0.00 \pm 0.00 \mathrm{i}$ & $0.00 \pm 0.00 \mathrm{e}$ \\
\hline Solenostemon scutellarioides 'Fuseables' & $0.40 \pm 0.08$ cdefghi & $0.03 \pm 0.02 \mathrm{de}$ \\
\hline Solenostemon scutellarioides Mariposa ${ }^{\mathrm{TM}}$ & $0.03 \pm 0.02 \mathrm{hi}$ & $0.03 \pm 0.02 \mathrm{de}$ \\
\hline Stachys byzantine & $0.02 \pm 0.02 \mathrm{hi}$ & $0.13 \pm 0.06$ bcde \\
\hline Thunbergia alata & $0.00 \pm 0.00 \mathrm{i}$ & $0.00 \pm 0.00 \mathrm{e}$ \\
\hline Tibouchina urvilleana & $0.02 \pm 0.02 \mathrm{hi}$ & $0.00 \pm 0.00 \mathrm{e}$ \\
\hline Verbena bonariensis & $0.10 \pm 0.04$ fghi & $0.04 \pm 0.02 \mathrm{de}$ \\
\hline Verbena canadensis 'Taylortown Red' & $0.15 \pm 0.06$ fghi & $0.05 \pm 0.03 \mathrm{de}$ \\
\hline Zea mays 'Tiger Cub' & $0.10 \pm 0.04$ fghi & $0.02 \pm 0.02 \mathrm{de}$ \\
\hline Zingiber malayensis & $0.00 \pm 0.00 \mathrm{i}$ & $0.00 \pm 0.00 \mathrm{e}$ \\
\hline
\end{tabular}

Means in the same column bearing different letters are significantly different $(\alpha=0.05)$.
(L.) Schott, among other plant species (Table 3).

Sweep-net sampling: Beneficial insects. Potential beneficial insect taxa observed included garden spiders (Araneae), soldier beetles (Cantharidae), predatory ants (Formicidae), fireflies (Lampyridae), predaceous plant bugs (Miridae i.e., Lygus sp.), preadatory damsel bugs (Nabidae), predaceous stink bugs (Pentatomidae i.e., Euthyrhynchus sp.), predatory tree crickets (Oecanthinae), bigeyed bugs (Geocoris punctipes), and parasitic wasps (Hymenoptera) (Table 4). These groups were combined into a single category: All Beneficial Insects. Plant taxa were constant, but the number of beneficial insects visiting each varied $(\mathrm{F}=2.98, \mathrm{df}=38,878$, $P=<0.0001$; Table 3).

Of the plant types sampled, the 15 plants where the most beneficial insects were collected were $R$. triloba, $B$. davidii, Nepeta $\times$ faassenii 'Walker's Low', C. spicata, Caryopteris $\times$ clandonensis Rehder 'First Choice', $S$. scrutellariodes 'Fuseables', S. microphylla 'Hot Lips', F. vulgare, A. millefolium 'Sunny Seduction', A. filipendulina 'Coronation Gold', Melissa officinalis L., R. hirta 'Indian Summer', Abelia $\times$ grandiflora 'Raspberry Profusion', $A$. hubrichtii, and Eupatorium purpureum ssp. maculatum (L.) E.E. Lamon 'Gateway' among other plant types (Table 3 ).

\section{Discussion}

To the best of our knowledge, this study is the first to document the response of pollinators and natural enemies to plantings of ornamental plants suitable for southeastern landscapes. One of the first steps toward conservation of bees and other beneficial insects is to determine which plants will provide adequate resources at different times in the growing season and can be implemented in landscape by land managers, landscape professionals, and homeowners (Tuell et al., 2008).

The gardens attracted a diverse population comprised of pollinators $(30+$ species and $16+$ families) and beneficial insects $(20+$ species and 9+ families). In addition, species of native bees [i.e., Lasioglossum (dialictus) imitatum Smith and H. ligatus] were identified in the gardens suggesting that pollinator habitats could be created in southeastern landscapes using these taxa. Celosia spicata, Gaura 'Passionate Blush', Lantana 'Mozelle' and 'Ms. Huff', and Nepeta $\times$ faassenii 'Walker's Low' were some of the most visited plants by both pollinators and beneficial insects. This could be due to the vibrant colors, rich nectar and pollen supply, and the variety of floral inflorescences these plants possess. Our research suggests nonnative exotic species, such as $C$. petiolata, $H$. acetosella, $S$. byzantine, and C. esculenta, may also be incorporated in landscape with potential to provide refuge and shelter for pollinators and beneficial insects.

Our study as well as previous research indicates the ecological value of plants are consistent for cultivar varieties and local, 
Table 3. Mean ( \pm SE) of pollinators and other beneficial insects collected via sweep net on plant types in the University of Georgia-Griffin Butterfly and Conservation Gardens $(n=4)$ (Spalding Co., GA).

\begin{tabular}{|c|c|c|}
\hline Plant name & All pollinators & All beneficial insects \\
\hline Abelia $\times$ grandiflora 'Raspberry Profusion' & $1.32 \pm 0.28 \mathrm{~b}$ & $0.96 \pm 0.23 \mathrm{bc}$ \\
\hline Achillea filipendulina 'Coronation Gold' & $1.36 \pm 0.59 \mathrm{~b}$ & $1.08 \pm 0.45 \mathrm{bc}$ \\
\hline Achillea millefolium 'Sunny Seduction' & $1.52 \pm 0.43 \mathrm{ab}$ & $1.09 \pm 0.23 \mathrm{bc}$ \\
\hline Agastache 'Black Adder' & $0.76 \pm 0.24 b$ & $0.41 \pm 0.24 \mathrm{bc}$ \\
\hline Amsonia hubrichtii & $1.75 \pm 0.29 \mathrm{ab}$ & $0.82 \pm 0.19 \mathrm{bc}$ \\
\hline Aster dumosus 'Wood's Pink' & $0.50 \pm 0.20 \mathrm{~b}$ & $0.75 \pm 0.41 \mathrm{bc}$ \\
\hline Astilbe chinensis 'Visions in Pink' & $1.08 \pm 0.38 \mathrm{~b}$ & $0.71 \pm 0.19 \mathrm{bc}$ \\
\hline Belamcanda chinensis & $1.22 \pm 0.36 \mathrm{~b}$ & $0.77 \pm 0.20 \mathrm{bc}$ \\
\hline Buddleia davidii 'Sweet Profusion' & $0.81 \pm 0.25 \mathrm{~b}$ & $2.81 \pm 0.70 \mathrm{ab}$ \\
\hline Caryopteris $\times$ clandonensis 'First Choice' & $0.50 \pm 0.50 \mathrm{~b}$ & $2.25 \pm 1.03 \mathrm{abc}$ \\
\hline Cassia $\times$ floribunda & $0.72 \pm 0.24 b$ & $0.72 \pm 0.24 \mathrm{bc}$ \\
\hline Celosia spicata & $3.89 \pm 1.12 \mathrm{a}$ & $1.96 \pm 0.48 \mathrm{abc}$ \\
\hline Cephalanthus occidentalis & $0.64 \pm 0.20 \mathrm{~b}$ & $0.80 \pm 0.18 \mathrm{bc}$ \\
\hline Chrysanthemum 'Cambodian Queen' & $0.81 \pm 0.21 \mathrm{~b}$ & $0.76 \pm 0.18 \mathrm{bc}$ \\
\hline Colocasia esculenta 'Black Stem' & $0.96 \pm 0.28 \mathrm{~b}$ & $0.50 \pm 0.14 \mathrm{bc}$ \\
\hline Curcuma petiolata 'Emperor' & $1.07 \pm 0.28 \mathrm{~b}$ & $0.59 \pm 0.22 \mathrm{bc}$ \\
\hline Dicliptera suberecta & $0.33 \pm 0.19 \mathrm{~b}$ & $0.47 \pm 0.19 \mathrm{bc}$ \\
\hline Echinacea purpurea 'Magnus' & $1.12 \pm 0.27 \mathrm{~b}$ & $0.76 \pm 0.28 \mathrm{bc}$ \\
\hline Eupatorium purpureum ssp. maculatum 'Gateway' & $0.68 \pm 0.24 \mathrm{~b}$ & $0.82 \pm 0.15 \mathrm{bc}$ \\
\hline Foeniculum vulgare & $0.62 \pm 0.22 \mathrm{~b}$ & $1.29 \pm 0.55 \mathrm{bc}$ \\
\hline Gaura lindheimeri 'Passionate Blush' & $0.45 \pm 0.23 \mathrm{~b}$ & $0.75 \pm 0.28 \mathrm{bc}$ \\
\hline Hedychium coronarium & $0.68 \pm 0.21 \mathrm{~b}$ & $0.32 \pm 0.11 \mathrm{bc}$ \\
\hline Heliopsis helianthoides var. scabra 'Summer Sun' & $0.26 \pm 0.10 \mathrm{~b}$ & $0.32 \pm 0.13 \mathrm{bc}$ \\
\hline Hibiscusacetosella Mahagony Splendor & $0.75 \pm 0.27 \mathrm{~b}$ & $0.54 \pm 0.18 \mathrm{bc}$ \\
\hline Lantana camara 'Mozelle' & $0.33 \pm 0.14 \mathrm{~b}$ & $0.50 \pm 0.23 \mathrm{bc}$ \\
\hline Lantana camara 'Ms. Huff' & $0.90 \pm 0.27 \mathrm{~b}$ & $0.75 \pm 0.18 \mathrm{bc}$ \\
\hline Lantana camara 'Pink Caprice' & $0.20 \pm 0.14 \mathrm{~b}$ & $0.13 \pm 0.09 \mathrm{c}$ \\
\hline Lysimachia ciliata 'Firecracker' & $2.21 \pm 0.58 \mathrm{ab}$ & $0.79 \pm 0.19 \mathrm{bc}$ \\
\hline Melissa officinalis & $0.40 \pm 0.13 b$ & $1.05 \pm 0.39 \mathrm{bc}$ \\
\hline Monarda didyma 'Raspberry Wine' & $0.85 \pm 0.34 \mathrm{~b}$ & $0.65 \pm 0.25 \mathrm{bc}$ \\
\hline Nepeta $\times$ faassenii 'Walker's Low' & $1.75 \pm 0.32 \mathrm{ab}$ & $2.71 \pm 0.38 \mathrm{abc}$ \\
\hline Rudbeckia fulgida var. sullivantii 'Goldsturm' & $1.63 \pm 0.53 \mathrm{ab}$ & $0.52 \pm 0.12 \mathrm{bc}$ \\
\hline Rudbeckia hirta 'Indian Summer' & $1.69 \pm 0.58 \mathrm{ab}$ & $1.00 \pm 0.21 \mathrm{bc}$ \\
\hline Rudbeckia triloba & $1.36 \pm 0.78 \mathrm{~b}$ & $4.05 \pm 2.08 \mathrm{a}$ \\
\hline Ruellia brittoniana 'Purple Showers' & $0.50 \pm 0.18 \mathrm{~b}$ & $0.81 \pm 0.29 \mathrm{bc}$ \\
\hline Salvia microphylla 'Hot Lips' & $0.85 \pm 0.20 \mathrm{~b}$ & $1.33 \pm 0.33 \mathrm{bc}$ \\
\hline Solenostemon scutellariodes 'Fuseables' & $0.66 \pm 0.25 \mathrm{~b}$ & $1.44 \pm 0.70 \mathrm{abc}$ \\
\hline Verbena bonariensis & $0.36 \pm 0.17 \mathrm{~b}$ & $0.27 \pm 0.13 \mathrm{bc}$ \\
\hline Zinnia angustifolia $\times$ elegans 'Profusion Deep Apricot' & $0.44 \pm 0.18 \mathrm{~b}$ & $0.59 \pm 0.15 \mathrm{bc}$ \\
\hline
\end{tabular}

Means in the same column bearing different letters are significantly different $(\alpha 0.05)$.

Table 4. Influence of plant taxa on occurrence of pollinating and beneficial insect taxa collected via sweep net in the University of Georgia-Griffin Butterfly and Conservation Gardens ( $n=4)$ (Spalding Co., GA).

\begin{tabular}{lccc}
\hline Insect type & df & $P$ value & F value \\
\hline Long-legged fly & 38,878 & $<0.0001^{* * *}$ & 2.31 \\
Bumble bee & 38,878 & $<0.0001^{* * *}$ & 4.46 \\
Carpenter bee & 38,878 & $<0.0001^{* * *}$ & 3.60 \\
Tumbling flower beetle & 38,878 & $0.0022^{* *}$ & 1.81 \\
Small bee & 38,878 & $<0.0001 * * *$ & 3.95 \\
Hover fly & 38,878 & $<0.0001 * * *$ & 3.03 \\
Western honeybee & 38,878 & $0.0418^{*}$ & 1.45 \\
Clearwing moth & 38,878 & $0.0026^{* *}$ & 1.79 \\
Fiery skipper & 38,878 & $0.0004^{* *}$ & 2.00 \\
Buckeye butterfly & 38,878 & $0.0233^{*}$ & 1.53 \\
All Pollinators combined & 38,877 & $<0.0001 * * *$ & 3.27 \\
Spider & 38,877 & $0.0004 * * *$ & 0.77 \\
Solider beetle & 38,878 & $0.0109 * *$ & 2.01 \\
Predaceous plant bug & 38,878 & $<0.0001^{* * *}$ & 1.29 \\
Predatory damsel bug & 38,878 & $0.0192^{* *}$ & 4.42 \\
Predatory stink bug & 38,878 & $<0.0001^{* * *}$ & 1.13 \\
Big-eyed bug & 38,878 & $<0.0001^{* * *}$ & 0.89 \\
All Beneficial Insects combined & 38,877 & $<0.0001^{* * *}$ & 4.81 \\
\hline
\end{tabular}

*, **, ***Significant at $P<0.05,0.01$, or 0.001 , respectively.

wild-type plants (Poythress and Affolter, 2015). Using these data, recommendations can be provided on the applied use of flowering ornamental plants for pollinator

\section{Literature Cited}

Astegiano, J., F. Massol, M.M. Vidal, P.O. Cheptou, and P.R. Guimarães, Jr. 2015. The robustness of plant-pollinator assemblages: Linking plant interaction patterns and sensitivity to pollinator loss. PLoS One 10(2):E0117243.

Fiedler, A.K. and D.A. Landis. 2007. Attractiveness of Michigan native plants to arthropod natural enemies and herbivores. Environ. Entomol. 36(4):751-765.

Frankie, G.W., R.W. Thorp, M. Schindler, J. Hernandez, B. Ertter, and M. Rizzardi. 2005. Ecological patterns of bees and their host ornamental flowers in two northern California cities. J. Kans. Entomol. Soc. 78(3):227-246.

Fukase, J. and A. Simons. 2016. Increased pollinator activity in urban gardens with more native flora. Appl. Ecol. Environ. Res. 14(1):297-310.

Kearns, C.A. and D.W. Inouye. 1997. Pollinators, flowering plants, and conservation biology. Bioscience 47(5):297-307.

Kremen, C., N. Williams, M. Aizen, B. GemmillHerren, G. LeBuhn, R. Minckley, L. Packer, and S. Potts. 2007. Pollination and other ecosystem services produced by mobile organisms: A conceptual framework for the effects of land-use change. Ecol. Lett. 10(4):239-314.

Lázaro, A. and Ø. Totland. 2014. The influence of floral symmetry, dependence on pollinators and pollination generalization on flower size variation. Ann. Bot. (Lond.) 114(1):157-165.

Matteson, K.C., J.S. Ascher, and G.A. Langellotto. 2008. Bee richness and abundance in New York City urban gardens. Ann. Entomol. Soc. Amer. 101(1):140-150.

McIntyre, N.E. and M.E. Hostetler. 2001. Effects of urban land use on pollinator (Hymenoptera: Apoidea) communities in a desert metropolis. Basic Appl. Ecol. 2(3):209-218

Nicholls, C.I. and M.A. Altieri. 2013. Plant biodiversity enhances bees and other insect pollinators in agro-ecosystems. A review. Agron. Sustain. Dev. 33(2):257-274.

NRC National Research Council. 2007. Status of pollinators in North America. Washington, DC. Nat. Acad. Pr. 307-308.

Pimentel, D. 2002. Encyclopedia of pest management, p. 1-931. 2nd ed. CRC Press, New York City, NY.

Poythress, J.C. and J.M. Affolter. 2015. Gardening for wildlife: Are native plant cultivars as effective as native plants propagated from local, wild populations for promoting native insect diversity. Acta Hort. 1085:487-492.

SAS Institute Version 9.2. 2010. SAS guide for personal computers, p. 1-429. 6th ed. SAS Institute, Cary, NC.

Tuell, J.K., A.K. Fiedler, D. Landis, and R. Isaacs. 2008. Visitation by wild and managed bees (Hymenoptera: Apoidea) to eastern U.S. native plants for use in conservation programs. Environ. Entomol. 37(3):707-718.

Wilde, H.D., K.J. Gandhi, and G. Colson. 2015. State of the science and challenges of breeding landscape plants with ecological function. Hort. Res. 2:1-8.

Woltz, J.M., R. Isaacs, and D.A. Landis. 2012. Landscape structure and habitat management differentially influence insect natural enemies in an agricultural landscape. Agric. Ecosyst. Environ. 152:40-49.

and beneficial insect conservation purposes as well as sampling methods that can be employed to effectively survey beneficial insect communities.
U.S. Fish and Wildlife Service. 2016. Pollinators Home Page. U.S. Fish and Wildlife Service, Washington, DC. 1 Mar. 2016. <http://www. fws.gov/pollinators/>. 